\title{
Babies and brains: habituation in infant cognition and functional neuroimaging
}

\section{Nicholas B. Turk-Browne*, Brian J. Scholl and Marvin M. Chun}

Department of Psychology, Yale University, New Haven, USA

\section{Edited by:}

Silvia A. Bunge, University of California

Berkeley, USA

Reviewed by:

Leslie J. Carver, University of California,

San Diego, USA

Scott P. Johnson, University of

California, USA

*Correspondence:

Nicholas B. Turk-Browne, Department of Psychology, Yale University,

2 Hillhouse Avenue, New Haven CT 06511, USA. e-mail: nicholas.

turk-browne@yale.edu
Many prominent studies of infant cognition over the past two decades have relied on the fact that infants habituate to repeated stimuli - i.e. that their looking times tend to decline upon repeated stimulus presentations. This phenomenon had been exploited to reveal a great deal about the minds of preverbal infants. Many prominent studies of the neural bases of adult cognition over the past decade have relied on the fact that brain regions habituate to repeated stimuli-i.e. that the hemodynamic responses observed in $\mathrm{fMRI}$ tend to decline upon repeated stimulus presentations. This phenomenon has been exploited to reveal a great deal about the neural mechanisms of perception and cognition. Similarities in the mechanics of these two forms of habituation suggest that it may be useful to relate them to each other. Here we outline this analogy, explore its nuances, and highlight some ways in which the study of habituation in functional neuroimaging could yield novel insights into the nature of habituation in infant cognition - and vice versa.

Keywords: repetition attenuation, repetition suppression, repetition enhancement, fMRI adaptation, priming, implicit memory, novelty preferences, cognitive neuroscience

\section{INTRODUCTION}

An important property of the human mind is that novel information and repeated information are treated differently. In particular, repeated stimuli tend to receive progressively less processing, and this habituation manifests itself in many ways ${ }^{1}$. While the discovery of novelty-seeking behavior extends far back into the history of psychological science, our goal in this paper is to explore the relationship between two particular manifestations that have been exploited in rather different subfields of cognitive science: studies of infant perception and cognition based on novelty preferences in looking time, and studies of adult perception and cognition based on repetition attenuation as measured with functional neuroimaging. The first two sections of this paper selectively review these two varieties of habituation, and the third section notes a nuanced set of similarities between them. The final section then explores ways in which the study of habituation in functional neuroimaging could yield novel insights into the nature of habituation in infant cognition - and vice versa.

\section{HABITUATION IN INFANT COGNITION}

Infants tend to look longer at novel stimuli than at repeated stimuli (for a recent review, see Aslin, 2007) $)^{2}$. Initial studies in

${ }^{1}$ As will become clear below, we use the procedural term "habituation" in a general sense in this paper, abstracting away from how it has been operationalized in specific domains, and without any quantitative assumptions about how much repetition is required. Moreover, while habituation refers to the process underlying decreased responsiveness itself, most studies that employ habituation instead examine the consequences of this process for subsequent processing of novel and familiar stimuli.

${ }^{2}$ This general avoidance of repeated information and exploration of novel information can be observed in many other types of responses in infants, including heart rate, respiration, startle, and sucking, but here we primarily focus on patterns of looking times as the dependent measure. infant cognition were primarily interested in habituation per se as a measure of simple learning in the youngest infants (e.g. Kagan and Lewis, 1965). In such studies, a single stimulus was repeated several times across trials, and the change in time spent fixating on the stimulus over repetitions served as the measure of habituation. Later studies began employing habituation as a research tool to investigate infants' abilities to discriminate and remember stimuli (e.g. Fagan, 1970; Wetherford and Cohen, 1973; see also Bornstein, 1985; Cohen, 1976; Olson, 1976). Critically, this approach involves examining patterns of fixation for repeated vs. novel stimuli, rather than just the decrease in responsiveness to repeated stimuli alone. The visual paired comparison procedure was an early example of this approach: when presented with two complex visual patterns, infants looked longer at a new pattern compared to one that they had previously seen several times (Fantz, 1964). However, in this case it is unclear whether the increased looking reflects a preference for the novel stimulus or avoidance of the repeated stimulus. This result was nevertheless groundbreaking, as it indicated that infants could discriminate the two alternatives, and had memory for the repeated stimulus.

More standard habituation tasks have convincingly demonstrated novelty preferences by relying on a separate test phase. In such designs, infants are repeatedly familiarized to a single stimulus for a fixed amount of time (e.g. Fagan, 1972), or - because of differences in the rate of habituation across subjects - until their looking time is reduced to a criterion level (typically, when the mean looking time over the last three trials is less than $50 \%$ of the mean looking time of the first three trials; see Cohen and Gelber, 1975). After reaching this criterion, infants begin a test phase. Perhaps the simplest test involves comparing the looking time to the habituation stimulus (now repeated again during test) vs. the looking time to a novel stimulus that differs from the habituation stimulus along 
some dimension, such as stimulus orientation or face identity. If the infant represents this dimension, perceives the difference, and finds the difference to be salient, they will exhibit a novelty preference by looking longer at the novel stimulus (sometimes called dishabituation). The novelty preference is quantified as the amount of extra time that the infant fixated the novel stimulus compared to the familiar stimulus, across trials. Since any systematic preference of this sort would only be possible if the infant can discriminate the two stimuli, this technique has been used extensively to describe the perceptual abilities of infants - for example revealing their ability to discriminate orientation (e.g. Atkinson et al., 1988) or faces (e.g. Young-Browne et al., 1977).

Novelty preferences have also been used to study other types of more complex perceptual and conceptual abilities. In many of these studies, the test phase consists of two stimuli that each differ from the familiarized stimulus along some of the same perceptual dimensions, but differ from each other along a critical additional dimension. Again, if infants discriminate this critical dimension, then they may look longer at the stimulus that is novel in this key respect. For example, this technique has been used to explore infants' understanding of simple physical principles - that objects exist even when unseen (the idea of "object permanence"), and cannot pass through each other (e.g. Baillargeon, 1987; Baillargeon et al., 1985; Spelke et al., 1992).

In one such study (Baillargeon, 1987), young infants were familiarized to a display in which a screen lying flat and facing the infant rotated up and away from the infant $180^{\circ}$ in the manner of a drawbridge (Figure 1A). This motion repeated until a habituation criterion was reached. At test, a new object was then placed at the far edge of the screen, which either did or did not interact with the rotating screen. In "impossible" test displays, the screen moved exactly as during familiarization, appearing to rotate right through the object. In "possible" test displays, the screen rotated only to the point where adults would expect it to contact the object and stop moving. Thus, both test displays were novel (containing a new object), but the "possible" event was more perceptually novel (containing a different screen rotation). Thus, if infants exhibit a perceptual novelty preference, they should look longer at the possible event. However, if they can discriminate the more abstract change (i.e. that the screen violated the principle of object permanence), and if they find this abstract change to be especially salient, then their looking times should increase to the impossible event. Indeed, infants looked longer at the "impossible" test trials (Figure 1B) which was interpreted in terms of a violation of infants' expectations regarding solidity and/or object permanence.

Similar violation-of-expectation studies using looking-time measures have explored many cognitive abilities of preverbal infants, in some cases without using a separate familiarization phase (e.g. Baillargeon, 2000; Wang et al., 2004). For example, one such study examined infants' simple arithmetic abilities (Wynn, 1992). In an "addition" condition, infants were presented with a single familiarization event in which a doll appeared on a stage, a screen
A Experimental Condition

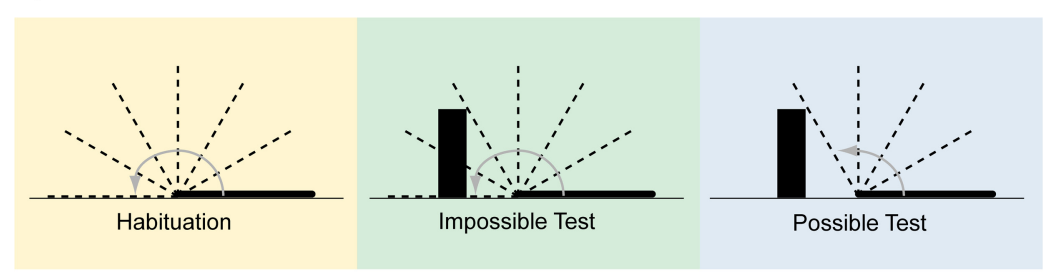

Control Condition
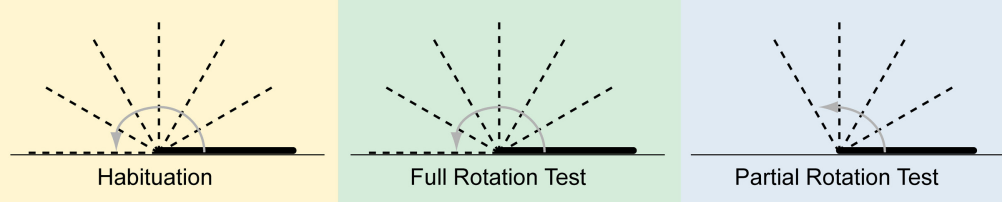
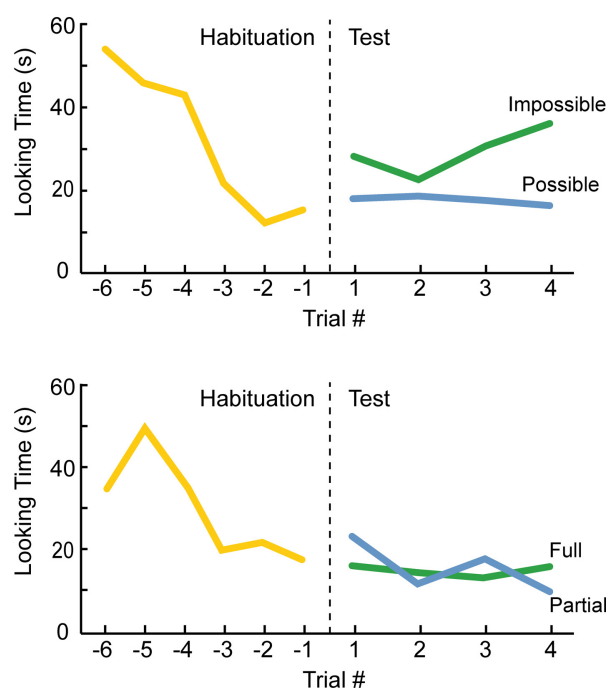

FIGURE 1 |An example of habituation in infant cognition (adapted from Baillargeon, 1987). (A) Side view of habituation and test displays. In both conditions, infants were habituated to a $180^{\circ}$ drawbridge-like motion. The decline and plateau of looking times during habituation are depicted in the left panels of (B). In the Experimental Condition, infants completed two types of test trials, both of which contained a new object on the far side of the display (depicted by the black box). The Impossible Test involved the same full $180^{\circ}$ rotation from habituation, but now the screen surprisingly passed through the box as it completed its rotation (with the box disappearing as it became obscured). The Possible Test involved a novel shorter rotation of screen up to the point where it would contact the box, where it stopped; this motion was "possible" in terms of solidity and object permanence, but was more perceptually nove/ than the Impossible Test. In the Control Condition, the screen rotations were identical, but no new object was presented (such that both motions were equally possible). Within each condition, the two types of test trials were alternated, with order counterbalanced across infants. The results from the test phase are depicted in the right panels of (B). In the Experimental Condition, infants dishabituated to the Impossible Test but not the Possible Test. However, in the Control Condition no preference was observed. These results were interpreted as reflecting the violation of infants' expectations regarding solidity and/or object permanence. 
rotated up and covered part of the stage, then another doll was placed behind the screen. The screen was then removed to reveal either one or two dolls on the stage. Infants looked longer at the single doll display, which was interpreted in terms of the arithmetic expectation that $1+1=2$. Similar evidence of arithmetic competence was found with $1+1=2$-or- 3 and $2-1=1$-or- 2 conditions. There has been substantial debate about how these studies that employ limited familiarization (e.g. Wynn, 1992) relate to those in which infants are habituated to criterion (e.g. Baillargeon, 1987). In particular, it has been argued that looking times following limited familiarization may reflect perceptual familiarity preferences rather than conceptual novelty preferences (e.g. Bogartz et al., 1997; Cohen and Marks, 2002). Nevertheless, we mention violation-ofexpectation studies here because they do often involve a similar design with repeated vs. novel displays.

These studies collectively illustrate the power of habituation and novelty preferences as phenomena that have been exploited to make inferences about infants' perceptual and cognitive abilities.

\section{HABITUATION IN FUNCTIONAL NEUROIMAGING}

Neural responses tend to be stronger to novel information than to repeated information (for recent reviews, see Grill-Spector et al.,
2006; Krekelberg et al., 2006; Schacter et al., 2007). This effect was initially observed in terms of reduced firing rates in single unit recordings from macaque inferior temporal cortex (e.g. Baylis and Rolls, 1987; Brown et al., 1987; Li et al., 1993; Miller and Desimone, 1994), but we will largely focus here on analogous effects observed in functional magnetic resonance imaging (fMRI) of human adults the phenomenon of repetition attenuation. In typical studies of rapid repetition attenuation (often called fMR-adaptation), each trial contains two images presented 300-500 ms apart. Following the presentation of the first image - which serves to familiarize the observer to the stimulus - the second image is either identical to the first image (repeated) or different along some dimension under investigation (novel). For example, the two images may have identical or different local contours (Figure 2A). Given the poor temporal resolution of fMRI, the aggregated hemodynamic response to both images is then analyzed. Repetition attenuation can then be quantified as the difference in the hemodynamic response between trials containing identical vs. different images.

Because fMRI typically provides data from many regions in the brain, we can consider how brain regions differ with respect to the amount of repetition attenuation they exhibit. In the example depicted in Figure 2A, if a population of neurons in one region does
A
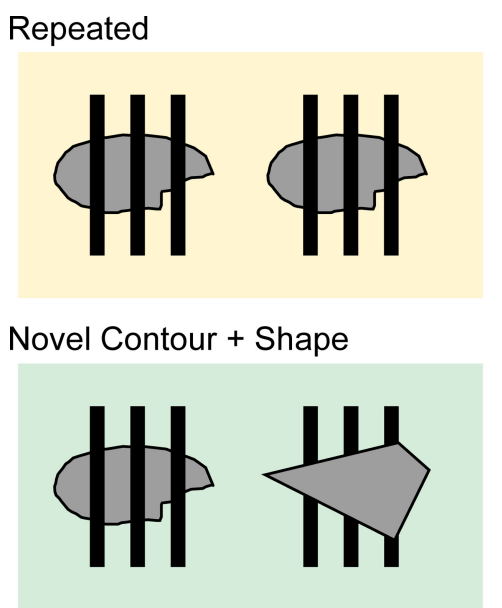

Novel Contour

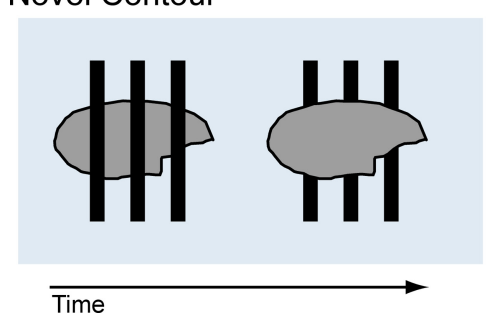

\section{Lateral Occipital Complex}

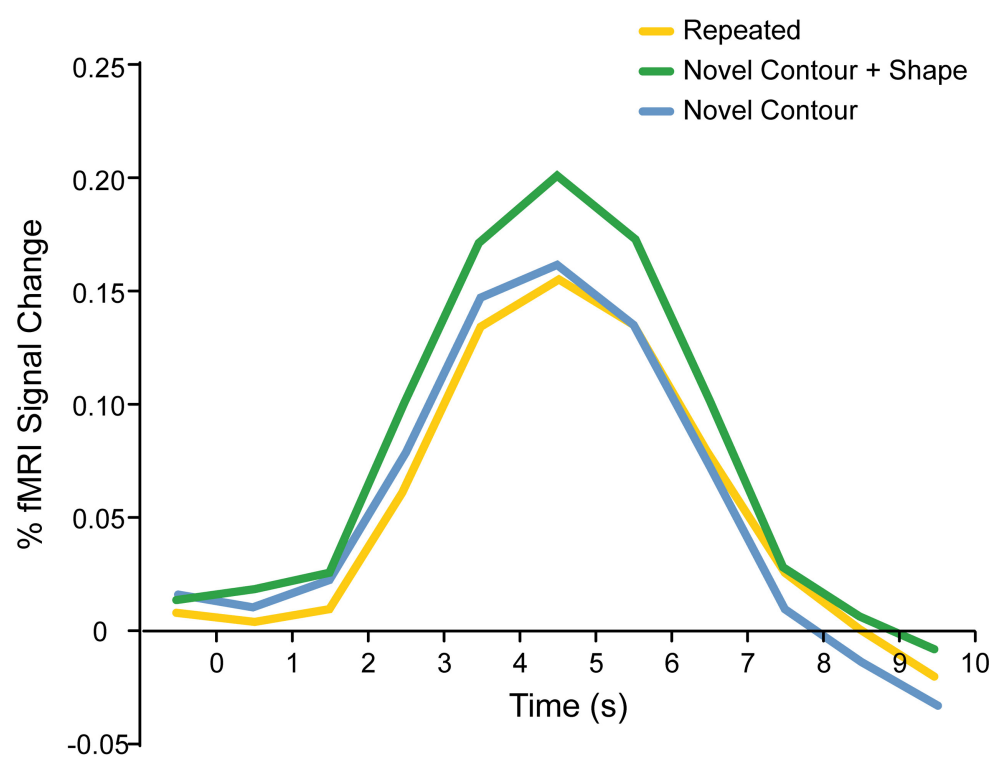

FIGURE 2 | An example of habituation in functional neuroimaging (adapted from Kourtzi and Kanwisher, 2001). (A) In each trial, two stimuli were presented sequentially (each for $300 \mathrm{~ms}$ with a $400 \mathrm{~ms}$ pause in between). The stimuli were simple shapes presented behind or in front of three partially occluding bars. In the Repeated trials, the same shape was repeated at the same depth with respect to the occluders. In the Novel Contour trials, the same shape was repeated, but moved in depth with respect to the occluders, such that the local visible contours changed. In the Novel Contour + Shape trials, a different shape was presented as the second stimulus, also moved in depth with respect to the occluders. (B) Event-related $\mathrm{fMRI}$ responses to the pairs of stimuli in a region of ventral visual cortex that responds more strongly to objects than to other stimulus patterns (lateral occipital complex; LOC). The response to the Novel Contour trials was the same as the response to the Repeated trials, demonstrating that the LOC does not represent visible local contours per se. However, the responses to both of these conditions were attenuated relative to the response to the Novel Contour + Shape condition. These results suggest that the LOC represents perceived shape rather than local contours. 
not represent local contours (such that it cannot discriminate two images on the basis of these contours), the fMRI response in this region will be the same for trials containing identical vs. different contours, and thus there will be no repetition attenuation. The same neuronal population may represent some other property of the stimuli (such that, for example, it can discriminate between two images on the basis of perceived shape); in these cases, the fMRI response in this region will be reduced for trials containing identical vs. different shapes, and thus exhibit repetition attenuation. Both of these patterns are apparent in Figure 2B (adapted from Kourtzi and Kanwisher, 2001).

Repetition attenuation is not an all-or-none affair, of course: the amount of attenuation within a particular brain region is proportional to the degree of selectivity along a given dimension and the magnitude of the difference between two stimuli along that dimension. For example, representations of objects in the lateral occipital complex (LOC; Malach et al., 1995) are orientationspecific: rotating an identical object $15^{\circ}$ elicits a greater fMRI response (i.e. less repetition attenuation) than if the object had been repeated in its original orientation, but rotating an identical object $45^{\circ}$ elicits an even greater response (Murray and Wojciulik, 2004). Similar parametric methods have also been used to study aspects of cognition, for example revealing a representation of approximate number in intraparietal sulcus (Piazza et al., 2004), and revealing a representation of how similar another person is to oneself in ventromedial prefrontal cortex (Jenkins et al., 2008).

Repetition attenuation is a prevalent consequence of stimulus repetition, and can be observed in modalities other than vision (e.g. Bergerbest et al., 2004; Dehaene-Lambertz et al., 2006), and in various brain regions (e.g. Breiter et al., 1996; Jenkins et al., 2008; Thompson-Schill et al., 1999). Moreover, it is not limited to situations in which the repeated stimulus is presented immediately following the initial stimulus. In fact, attenuated hemodynamic responses to repeated stimuli can be observed after one or more interleaved novel stimuli (e.g. Turk-Browne et al., 2006), or after significant interference (e.g. Henson et al., 2004) and delays (van Turennout et al., 2000). In these cases, hemodynamic responses are individually estimated for the repeated stimulus (rather than the pair, as in the rapid repetition case described above) and compared to the hemodynamic responses for novel stimuli that are presented during that same phase of the experiment. However, note that repetition attenuation is not universal, and many factors contribute to whether or not it will be observed, including selective attention (e.g. Yi and Chun, 2005), task context (e.g. Dobbins et al., 2004; cf. Xu et al., 2007) and explicit recognition (e.g. Schott et al., 2005). Thus, repetition attenuation is most readily observed when stimuli are attended, but repetitions can nevertheless be incidental to the task at hand (e.g. during orthogonal categorization judgments; Dobbins et al., 2004; Turk-Browne et al., 2006).

These examples collectively illustrate the power of habituation in adult cognitive neuroscience as a phenomenon that can be exploited to make inferences about the nature of perceptual and cognitive representations.

\section{THE ANALOGY}

The two types of habituation described above are superficially similar, each reflecting a type of decreased responsiveness to repeated stimuli. In the case of infant cognition, infants may be repeatedly familiarized to a stimulus until a preset habituation criterion is met. They are then presented with test stimuli that are more or less similar to the familiarized stimulus in various ways. Lookingtime preferences for novel stimuli over repeated stimuli serve as the primary dependent measure, and indicate both that the infant can discriminate along the dimension that distinguishes the test items, and that they have memory for the repeated stimulus. In the case of functional neuroimaging, observers are familiarized to an initial stimulus (typically once, but up to three or more times; e.g. Dobbins et al., 2004; Grill-Spector et al., 1999). They are then presented with a second test stimulus that is more or less similar to the initial stimulus. Attenuated hemodynamic responses within a brain region for repeated stimuli serve as the primary dependent measure, and indicate both that the brain region can discriminate the test items, and that it has represented the repeated stimulus. Thus, both novelty preferences and repetition attenuation reflect increased processing of new information ${ }^{3}$.

Given that these two subfields of cognitive science have been using similar phenomena as tools to explore similar issues, it is unfortunate that they have typically been treated in isolation. To our knowledge, previous explorations of infant habituation have almost never discussed fMRI repetition attenuation, and vice versa. We think, however, that a theoretical comparison of these two literatures is instructive, since the analogies run deep. Below we review three of these analogies that seem particularly useful to consider in the context of relating these two literatures. It is possible that these analogies suggest a common underlying mechanism for these two forms of habituation, but we will argue that comparing these two literatures is of considerable use and interest even if different but analogous mechanisms are involved.

\section{MEASUREMENT SENSITIVITY}

A first similarity worth mentioning relates to the development of each method: these forms of habituation have each achieved their prominence largely as a result of the increased sensitivity they afford over other methods. This is especially salient in infant cognition, where looking-time measures proved more sensitive than previous methods based on overt action and manipulation. For example, Piaget (1954) famously suggested that object permanence did not develop until 18-24 months, as indicated by younger infants' failures to search for hidden objects - but this implication was belied by later looking-time experiments, such as those described above. Looking-times, in other words, seem to constitute a better measure of underlying competence, and "it is no exaggeration to say that without looking time measures, we would know very little about nearly any aspect of infant development" (Aslin, 2007, p. 48).

Habituation methods in functional neuroimaging have similarly become prominent in part because they afford a degree of sensitivity that is difficult to obtain with other methods. For example,

${ }^{3}$ The interpretation of habituation data can depend critically on the stage of development, ranging from simple perceptual abilities that can affect how well a habituation stimulus is resolved, to more advanced cognitive abilities such as working memory and language that may support or confound performance in a particular experimental protocol. Note, however, that in this section we focus on theoretical analogies, rather than more specific developmental comparisons of, for example, how fast or to what properties infants vs. adults habituate. 
due to limitations in spatial resolution, traditional neuroimaging methods average over the fine-grained representational structure of visual cortex (e.g. Hubel and Wiesel, 1977; Tanaka, 1996). However, repetition-based methods afford higher functional resolution: if habituation is observed within a particular voxel when a stimulus is repeated, one can infer that a subpopulation of neurons within this region is tuned to the stimulus. For example, in an fMRI study of face processing (Winston et al., 2004), conventional localizer scans demonstrated that both the fusiform face area (FFA; Kanwisher et al., 1997; McCarthy et al., 1997) and the anterior superior temporal sulcus respond more strongly to faces than to other objects - and are thus face-selective in a classical sense-but repetition attenuation revealed that these areas process different aspects of faces: the FFA exhibited repetition attenuation when the identity of a face was repeated vs. changed, suggesting that this region represents facial identities - but the STS exhibited repetition attenuation when the expression of a face was repeated vs. changed, suggesting that this region represents different facial expressions.

Note that this example also illustrates the sensitivity of repetition attenuation methods in a different way, since it would not be possible to obtain such results without this phenomenon. It is true that, by using more classical subtraction techniques, one could simply contrast blocks of neutral faces with blocks of emotional faces. This would yield activation in the fusiform to both blocks (perhaps relative to fixation or to another category of stimuli such as line-drawings or houses), and enhanced activation in the STS for the emotional vs. neutral faces. One could then (unjustifiably) interpret these results as demonstrating that the fusiform is involved in processing identity and that the STS is involved in processing expression. However, repetition designs provide more specific information about both regions. (1) In the fusiform, observing activation for blocks of faces may simply reflect processing of different facial features, or even more generic holistic processing. To isolate "identity" one must titrate stimulus conditions such that they differ only with respect to the identity dimension - as when repeating blocks of same faces and blocks of different faces, and analyzing the resulting differential repetition attenuation (GrillSpector et al., 1999). One can even divorce identity from perceptual similarity by combining repetition attenuation with face-morphing techniques (e.g. Rotshtein et al., 2005). (2) In the STS, increased activation for emotional faces vs. neutral faces may simply reflect selectivity for any non-neutral facial expression, or more generic processing of valenced stimuli (e.g. yielding equal activation for blocks of angry faces vs. blocks of fearful faces). However, a repetition approach can demonstrate that the STS codes for particular facial expressions, by demonstrating dishabituation to an angry face following a fearful face, compared to a trial containing two fearful faces (e.g. Winston et al., 2004).

As we stress the enhanced sensitivity afforded by habituation techniques in infant cognition and adult cognitive neuroscience, note that it is not simply that such methods in isolation are more sensitive than some other methods. Rather, the particular questions that they can answer are analogous: in both domains, habituation techniques provide a way of indirectly probing for knowledge. This is particularly important in infant cognition, for both historical and scientific reasons: when other performance measures failed to elicit effects, theorists were led to underestimate infants' surprising competence in some areas. Repetition attenuation in fMRI has also proven important for characterizing the multifaceted nature of visual representations, which are otherwise aggregated into overt behavioral responses and/or obscured in classical fMRI analyses. Thus, in both cases, habituation techniques provide more specific information about types of knowledge $e^{4}$.

\section{MEMORY AND INTERRUPTIBILITY}

The visual environment is inherently dynamic, and we rarely reencounter the same stimulus without interruption by other stimuli, or after some delay in time. This fact has motivated research in infant cognition and functional neuroimaging that attempts to draw the connection between habituation and memory. In functional neuroimaging, attenuation can be observed to a repeated stimulus despite multiple intervening stimuli which themselves elicit normal (i.e. unhabituated) responses, even over long delays (van Turennout et al., 2000) and even when the interruption contains other examples from the same category (e.g. Buckner et al., 1998; Dobbins et al., 2004; Henson et al., 2000; Turk-Browne et al., 2006). This long-lag habituation has been used to study the neural basis of repetition priming, a form of implicit memory (e.g. Henson, 2003; Schacter and Buckner, 1998; Schacter et al., 2007; Wiggs and Martin, 1998). In particular, habituation in some brain regions is accompanied by decreases in response time (e.g. Dobbins et al., 2004; Turk-Browne et al., 2007), positively correlates with the degree of priming (e.g. Maccotta and Buckner, 2004; Turk-Browne et al., 2006), and may even be causally necessary for priming to occur (Wig et al., 2005). Thus, habituation may reflect the encoding of long-term perceptual memory (Turk-Browne et al., 2006; Yi and Chun, 2005).

Habituation of looking times in infant cognition may also reflect a form of implicit perceptual memory (see Nelson, 1995) ${ }^{5}$, with decreased looking time to familiar stimuli persisting across delays (e.g. Cohen et al., 1977). Moreover, infants' representations of habituated stimuli can persist despite interruptions due to interleaved

${ }^{4}$ Despite these benefits, note that habituation measures do not exhaust sensitivity Two stimuli that elicit similarly habituated responses - i.e. equally attenuated looking times or fMRI responses - may still be discriminable. For example, certain properties may be more or less salient to infants (leading them to look longer, or not) based on whether the particular test event is categorized as a type for which that property is relevant (Quinn, 1987; Wang et al., 2005). Thus a failure to dishabituate to the novel height of an object at test may indicate not that infants failed to notice the difference, but that they did not consider it relevant (Wang and Baillargeon, 2006). The same is true in functional neuroimaging. For example, just because a given brain region (say, the FFA) habituates without regard for some property (say, facial expression), that property may nevertheless be discriminated both behaviorally and in other brain regions, and other methods could even still reveal sensitivity to that property in that same brain region (cf. Kourtzi and Huberle, 2005; Murray et al., 2006; Park et al., 2007).

${ }^{5}$ While novelty preferences are believed to rely on the hippocampus (McKee and Squire, 1993), and thus would typically be considered a form of explicit or declarative memory, looking-time preferences (following habituation or in a paired-comparison task) can be observed much earlier in development than explicit recognition or recall in other tasks. These results among others have led to speculation that orienting to novelty is obligatory in young infants, and such automaticity is certainly more characteristic of implicit than explicit memory (Nelson, 1995). As further evidence, a recent study of adults demonstrated a doubledissociation between novelty preferences in looking time and explicit recognition, with classical conditioning affecting novelty preferences but not recognition memory, and stimulus complexity affecting recognition memory but not novelty preferences (Snyder et al., 2008; cf. Manns et al., 2000). 
stimulus patterns (e.g. Cohen et al., 1977; Quinn, 1987), display manipulations such as the arithmetic events described earlier (e.g. Feigenson et al., 2002; Wynn, 1992), and even extrinsic interruptions involving novel objects and events (Cheries et al., 2006). These illustrations of successful habituation across delays and interruptions suggest that habituation is well tuned to our highly complex and dynamic visual environment.

\section{FAMILIARITY: ATTENUATION VS. ENHANCEMENT}

One of the most intriguing analogies between habituation in these two domains relates to an oversimplification in the previous discussion. In our initial descriptions of habituation in both infant cognition and functional neuroimaging, we noted that repeated stimuli tend to elicit novelty preferences and repetition attenuation, respectively, but in fact this is not always the case. Indeed, some looking-time studies with infants yield familiarity preferences - i.e. longer looking at familiar vs. novel events (e.g. Bogartz et al., 1997, 2000; Cashon and Cohen, 2000; Cohen and Marks, 2002; Hunt, 1970; Richards, 1997; Roder et al., 2000; Wetherford and Cohen, 1973). For example, when infants are briefly familiarized to a display with one toy on a stage, they look longer at a test display containing one toy than at a test display containing two toys (Cohen and Marks, 2002). Similarly, some fMRI studies of adult visual cognition give rise to repetition enhancement - i.e. a stronger fMRI signal to repeated vs. novel events (e.g. Dolan et al., 1997; Grill-Spector et al., 2000; Henson et al., 2000; James and Gauthier, 2006; Kourtzi et al., 2005; Turk-Browne et al., 2007). For example, the repetition of two unfamiliar faces or line drawings can lead to enhanced responses in right fusiform cortex (Henson et al., 2000).

In some cases, this difference may not be theoretically important. For example, if the goal of a study simply to determine whether infants (or a brain area) can discriminate two stimuli, then a differential response in either direction is sufficient (as is the case for many other measures, e.g. Fiser and Aslin, 2002). In other contexts, however, this difference is critical - especially in infant cognition. For example, the interpretation of the study of infants' arithmetic abilities described above (Wynn, 1992) depends on the assumption of a novelty preference: when infants see $1+1=2$, their resulting longer looking at one object is taken to reflect a violation of expectation rather than a mere familiarity preference (Cohen and Marks, 2002). Because familiarity preferences have been observed, however, these interpretations have led to lively debates about the proper interpretation of looking-time data. Some researchers claim that the relevant data can be explained without appeal to "initial knowledge" of the world, based on familiarity preferences (e.g. Bogartz et al., 1997; Cohen and Marks, 2002; Haith, 1998). Others have attempted to rebut these arguments, arguing that these interpretations cannot account for the full array of data in each domain (e.g. Baillargeon, 1999; Carey, 2002; Spelke, 1998; Wynn, 2002).

Familiarity preferences and repetition enhancement are not commonly observed, and are seen in each literature as an exception to a general rule. Yet, because of the interpretive difficulties noted above, it is obviously important to determine just how and when familiarity vs. novelty preferences (repetition enhancement vs. attenuation) will arise. And strikingly, the answers here may be the same in both domains. In the most general terms, repeated stimuli may receive enhanced processing until they have been fully represented; at some point, diminishing returns may redirect processing efforts to novel stimuli. It follows then that the response to a stimulus that has been weakly represented should be enhanced relative to a novel stimulus, while the response to a stimulus that has been robustly represented should be attenuated relative to a novel stimulus. Such effects have been demonstrated in both infant cognition and functional neuroimaging.

In infant cognition, the likelihood of observing familiarity preferences vs. novelty preferences depends on a variety of factors, including age, stimulus complexity, exposure time, processing speed, and task difficulty (Hunter and Ames, 1988). These factors can all be summarized under a single organizing principle: preference depends on the quality of the representation established by past experience. This point can be made especially clear by considering how the quantity of habituation affects preferences: infants that are fully habituated to a stimulus exhibit novelty preferences, while infants whose habituation has been interrupted exhibit familiarity preferences (e.g. Hunter et al., 1982; Roder et al., 2000; Rose et al., 1982) ${ }^{6}$. Interestingly, the transition from familiarity to novelty preferences after sufficient habituation is not gradual: if habituation is interrupted immediately before infants would begin to show novelty preferences, they exhibit familiarity preferences of an equal magnitude to those at the beginning of habituation (Roder et al., 2000).

One might also expect from this discussion that during the process of a proper familiarization to criterion, infants would show an initial increase in looking time to the habituation stimulus, followed by a decline. Typically, however, habituation curves are monotonically decreasing. There are two potential reasons for the apparent lack of an initial increase. First, infants may exhibit generic decreases in responsiveness across trials that may mask an initial increase. In other words, looking time may decline between the first and second habituation trials, but nevertheless be enhanced relative to the looking time to a hypothetical novel stimulus. Second, averaging looking times over all infants in a sample may actually obscure initial increases. In other words, if some infants habituate immediately, while others habituate more slowly, any enhanced responses in the slow habituators would be obliterated by the already habituated responses of the fast habituators. To address this problem, several studies have aligned backwards from the point at which each infant reaches the habituation criterion (e.g. Cohen and Gelber, 1975; Roder et al., 2000). And consistent with the view sketched above, these studies observe that responses to familiar stimuli are enhanced immediately prior to reaching criterion (the point at which infants are exhibiting reliable novelty preferences). Interestingly, in adults, the transition from familiarity to novelty preferences can occur quickly during online processing: during individual test trials of a visual paired comparison task, observers tend to look first at the familiar stimulus and subsequently at the novel stimulus (Snyder et al., 2008).

In functional neuroimaging, the likelihood of observing repetition enhancement vs. attenuation depends on several factors,

${ }^{6}$ Some studies have also observed increased looking to familiar stimuli after habituation to criterion (Bertenthal et al., 1983). However, such increases are judged relative to the most recent pre-criterion looking times, and are believed to reflect spontaneous regression. Thus, to assess true preferences, it is necessary to compare looking times for novel and familiar stimuli in the post-criterion phase only. 
including stimulus exposure time (Grill-Spector et al., 2000), image quality (Dolan et al., 1997), and discriminability (Kourtzi et al., 2005) - all of which plausibly affect the quality of the resulting representation. For example, in the study mentioned above in which unfamiliar faces and line drawings produced repetition enhancement, familiar versions of the same stimuli (famous faces or common symbols) produced repetition attenuation in the same brain region (Henson et al., 2000), presumably because robust representations of these familiar stimuli already existed. More directly, the amount of information that can be extracted from a single stimulus exposure determines whether enhancement or attenuation will be observed when the stimulus is first repeated. For example, in the parahippocampal place area (PPA; Aguirre et al., 1998; Epstein and Kanwisher, 1998), the repetition of low-visibility scene photographs elicited repetition enhancement, while the repetition of clearly visible scene photographs elicited repetition attenuation (Turk-Browne et al., 2007). Unlike looking-time habituation, the timecourse of the transition from repetition enhancement to repetition attenuation is unknown. This is an important question for future research, since null results in repetition designs may reflect an intermediate stage of habituation. Interestingly, after extensive habituation, repetition attenuation begins to saturate, and eventually decline (Zago et al., 2005).

Moreover, initial increases in responsiveness (sensitization) followed by sustained decreases (habituation) are commonplace in various animal behaviors, including hindlimb flexion in cats following skin shocks, the startle response in rats following loud tones, and skin conductance in humans also following loud tones (Groves and Thompson, 1970). In these cases, sensitization is proportional to the intensity of the stimulus, and reflects excitability of the evoked response. However, these effects are transient and non-specific to the habituating stimulus. What makes the analogy between infant looking times and hemodynamic responses particularly interesting is that the transition from enhancement to attenuation may occur for the same underlying reason (as described above), reflecting stimulus-specific learning. Since similar effects in other behaviors and physiological responses are not stimulusspecific, such an explanation could not apply.

In sum, enhanced responses to repeated stimuli seem to occur for the same types of reasons in both infant cognition and functional neuroimaging - and these similarities fuel speculation that common representational dynamics may be involved. While many results in the two literatures are consistent with these ideas, it will certainly be worth directly testing this hypothesis in future studies.

\section{EXPLORING THE ANALOGY}

The primary goal of this paper has been to highlight an analogy between certain phenomena, methods, results, and mechanisms from two very different literatures. This alone seems worthwhile: the points of connection between habituation as it is used in infant cognition and functional neuroimaging are remarkable, despite the fact that these two phenomena have not been previously connected in this way. But the hope is that this analogy could itself facilitate progress in each area, and serve as a novel type of case study of how neuroscience and infant cognition may usefully interact.

Perhaps the most obvious way to pursue this interaction would be to empirically compare and contrast looking time novelty preferences and fMRI repetition attenuation in the same population. At present, exploring fMRI repetition attenuation in young infants poses considerable methodological challenges, including generic problems of head-motion and loud noise in the scanner, as well as the likelihood that such factors would themselves influence looking-time responses. As technology advances and new methodologies are developed, however, it may become feasible to obtain reliable fMRI data from young infants - in which case it would be fascinating to explore neural responses in the infant brain to the same displays that have been used in looking-time studies ${ }^{7}$.

The alternative - obtaining looking-time data from adults - is also methodologically challenging, for equally important but more abstract reasons. The goal of such a comparison would be to study the same underlying processes in both infants and adults. In general, however, it is rarely possible to achieve this goal in different populations by employing identical stimuli; instead, studying the same processes in two populations often requires using very different displays and tools, since the same displays would bring online different types of processes and strategies for the different populations. This possibility is clear in the present context, since adults' looking times - especially to the kinds of simple displays that are characteristic of infant looking-time studies - would surely reflect many different processes beyond those active in infants. For example, when viewing such displays adults' looking times would be influenced by their higher-level questions about how the experimenters managed to create a seemingly-impossible event, what the experiment is testing, etc. As a result, studies of adult looking behavior in simple displays have not typically been able to obtain meaningful results from looking time per se, and have focused instead on where adults will first fixate upon initially encountering a possibly-repeated display (e.g. Manns et al., 2000; Snyder et al., 2008). More generally, the displays used in infant studies may suffice to attract infants' attention repeatedly, but more complex displays may be required to obtain meaningful looking time data from adults (e.g. Ryan et al., 2007). Thus, equating the same underlying processing in these populations might actually require quite different paradigms and displays.

Though comparisons of these two forms of habituation in the same population resist straightforward empirical testing, such comparisons can nevertheless suggest potentially useful future directions. We conclude by noting four such possibilities:

First, beyond the connections noted above, these literatures are each quite rich, and each contains many manipulations and questions that could be usefully imported into the other. In this way, the analogy could be of great heuristic use, by suggesting new questions for each domain. For example, the role of attention in habituation has been treated very differently in the two literatures. In functional neuroimaging, attention is manipulated via task demands and is typically characterized in terms of resources (e.g. Pessoa

\footnotetext{
${ }^{7}$ There is also a smaller literature on repetition effects in adults as measured by EEG (e.g. Henson et al., 2004; Puce et al., 1999). Such methods are in some cases more amenable to pediatric samples than fMRI, and thus may be well suited to examining neural repetition effects in infants (e.g. Southgate et al., 2008; Wiebe et al., 2006). Repetition effects of this sort may thus provide an additional measure of habituation and dishabituation in infants that can be related to looking times (e.g. Snyder and Keil, in press), and may also help researchers explore how perceptual representations change over development (e.g. Gliga and Dehaene-Lambertz, 2007).
} 
et al., 2002) and selection (e.g. O'Craven et al., 1999). Although fMRI habituation is relatively implicit and automatic - such that it can occur despite depleted resources (Yi et al., 2004) - selective attention is nevertheless required (Eger et al., 2004; Murray and Wojciulik, 2004; Vuilleumier et al., 2005; Yi and Chun, 2005). In infant cognition, attention is often characterized in terms of passive states of arousal, for example as measured by heart rates (e.g. Richards, 1997) and manipulated by feeding (e.g. Geva et al., 1999). These states of arousal affect the rate of habituation: after equivalent amounts of exposure, infants exhibit novelty preferences during sustained attention and after feeding, but familiarity preferences during casual attention and prior to feeding (Geva et al., 1999; Richards, 1997). It may be worthwhile to import these different notions of attention into each domain. For example, we know of no direct research in functional neuroimaging on the relationship between arousal and habituation. One promising approach may be to examine how low-frequency shifts in the fMRI signal - that are believed to reflect arousal (Chawla et al., 1999) and other cognitive states (Leber et al., 2008) - influence repetition attenuation (Turk-Browne et al., 2006).

Second, the exploration of this analogy could help to elucidate the underlying cognitive processes that are involved in mediating various types of habituation. In functional neuroimaging, for example, habituation has been interpreted with regard to different forms of memory depending on the lag between repetitions: attenuated responses to repetitions after minimal delays or interruption may reflect short-term transient changes in stimulus-specific processing, while attenuated responses to repetitions over significant delays and interruption may result from long-term learning (see Grill-Spector et al., 2006; Henson, 2003). In contrast, habituation in infant cognition has often been interpreted in terms of the exercise of explicit knowledge (e.g. Spelke, 1988) vs. implicit visual processing (e.g. Cheries et al., in press; Feigenson et al., 2002; Scholl and Leslie, 1999), and has been related to constructs from vision science, such as “object files" (e.g. Carey and Xu, 2001; Cheries et al., 2006; Chiang and Wynn, 2000; Feigenson et al., 2002; Scholl and Leslie, 1999). Thus, it could be of use in each area to consider explanations based on constructs from the other. For example, the finding that lookingtime habituation can persist across interruptions by a small number but not a larger number of novel moving objects was interpreted in terms of object files (Cheries et al., 2006), but could also be interpreted in terms of working memory processes, which are similarly capacity-limited. Likewise, while neural repetition effects decline as the lag between repetitions increases (Henson et al., 2004), no research has examined whether such effects are sensitive to the complexity of the intervening items per se.

Third, our understanding of habituation itself may be enhanced by considering the analogy, since different kinds of models have been developed for infant and neural habituation. For example,

\section{REFERENCES}

Aguirre, G. K., Zarahn, E., and D’Esposito, M. (1998). Neural components of topographical representation. Proc. Natl. Acad. Sci. U.S.A. 95, 839-846.

Aslin, R. N. (2007). What's in a look? Dev. Sci. $10,48-53$.

detailed computational models of infant habituation (e.g. Schoner and Thelen, 2006; Sirois and Mareschal, 2002) could perhaps be supplemented by the neurophysiological accounts of habituation and its consequences that have been invoked in functional neuroimaging, including notions of fatigue (as often invoked in infant habituation), but also learning-related representational changes that result in speeded processing and increased selectivity (see Desimone, 1996; Grill-Spector et al., 2006; Wiggs and Martin, 1998). In particular, increased selectivity and hence reduced neuronal responses may bias competition for limited processing resources to novel stimuli, which evoke larger responses (Desimone and Duncan, 1995; cf. Snyder et al., 2008). In turn, the neurophysiological mechanisms underlying repetition attenuation are relatively unknown (e.g. Sawamura et al., 2006), but may depend heavily on competitive interactions between neurons that are initially broadly-tuned (see Grill-Spector et al., 2006); such ideas, often invoked at the level of mental representations, are an important component of models of infant habituation and development (e.g. Johnson, 2000; Westermann et al., 2007). Thus, an integration of models in the two domains could help us understand the dynamics of habituation in each case.

Fourth, it remains possible that that these two forms of habituation, despite their differences, may reflect the same types of underlying processes. In this case, exploring the analogy noted here could lead not only to progress in each area due to the heuristic consideration of ideas and models from the other area, but could perhaps unite these two domains. To accomplish this, both forms of habituation could be used as tools to study the same topics, with the goal of evaluating how similar the results are. For example, stimulus identity in functional neuroimaging (especially of ventral cortex) is typically evaluated in terms of featural and categorical information, whereas identity in infant cognition has often been evaluated with regard to spatiotemporal continuity (regardless of surface features; see Flombaum et al., in press). The analogy noted here, however, suggests that it might thus be useful to investigate both of these ideas with fMRI habituation. And when this was actually done, in fact, the FFA and the LOC were sensitive to both types of "sameness": in dynamic motion displays, repetition attenuation was observed only when featurallyidentical faces were repeated along a spatiotemporally continuous path (Yi et al., 2008). This provides a new way to think about how featural and spatiotemporal information are combined.

It is our hope that attending to this analogy could help us understand the nature and dynamics of habituation in each field, and ultimately help us appreciate the meaning of these similarities across such different areas of cognitive science, as a type of case study of how neuroscience and infant cognition may be mutually informative.

\section{ACKNOWLEDGMENTS}

For helpful conversations and/or comments on earlier drafts, we thank Erik Cheries, Marcia Johnson, Koleen McCrink, and Steve Mitroff.

Baillargeon, R. (1999). Young infants' expectations about hidden objects: a reply to three challenges. Dev. Sci. 2, 115-163.

Baillargeon, R. (2000). Reply to Bogartz, Shinskey, and Schilling; Schilling; and Cashon and Cohen. Infancy 1, 447-462.
Baillargeon, R., Spelke, E. S., and Wasserman, S. (1985). Object permanence in five-month-old infants. Cognition 20, 191-208.

Baylis, G. C., and Rolls, E. T. (1987). Responses of neurons in the inferior temporal cortex in short term and serial recognition 
memory tasks. Exp. Brain Res. 65, 614-622.

Bergerbest, D., Ghahremani, D. G., and Gabrieli, J. D. E. (2004). Neural correlates of auditory repetition priming: reduced fMRI activation in the auditory cortex. J. Cogn. Neurosci. 16, 966-977.

Bertenthal, B. I., Haith, M. M., and Campos, J. J. (1983). The partial-lag design: A method for controlling spontaneous regression in the infantcontrol habituation paradigm. Infant. Behav. Dev. 6, 331-338.

Bogartz, R. S., Shinskey, J. L., and Schilling, T. H. (2000). Object permanence in five-and-a-half-month-old infants? Infancy 1, 403-428.

Bogartz, R. S., Shinskey, J. L., and Speaker, C. J. (1997). Interpreting infant looking: the event set $\mathrm{x}$ event set design. Dev. Psychol. 33, 408-422.

Bornstein, M. H. (1985). Habituation of attention as a measure of visual information processing in human infants: summary, systematization, and synthesis. In Measurement of Audition and Vision in the First Year of Postnatal Life: A Methodological Overview, G. Gottlieb and N. A. Krasnegor, eds (Norwood, NJ, Ablex), pp. 253-300.

Breiter, H. C., Etcoff, N. L., Whalen, P. J., Kennedy, W. A., Rauch, S. L., Buckner, R. L., Strauss, M. M., Hyman, S. E., and Rosen, B. R. (1996). Response and habituation of the human amygdala during visual processing of facial expression. Neuron 17, 875-887.

Brown, M. W., Wilson, F. A., and Riches, I. P. (1987). Neuronal evidence that inferomedial temporal cortex is more important than hippocampus in certain processes underlying recognition memory. Brain Res. 409, 158-162.

Buckner, R. L., Goodman, J., Burock, M., Rotte, M., Koutstaal, W., Schacter, D., Rosen, B., and Dale, A. M. (1998). Functional-anatomic correlates of object priming in humans revealed by rapid presentation event-related fMRI. Neuron 20, 285-296.

Carey, S. (2002). Evidence for numerical abilities in young infants: a fatal flaw? Dev. Sci. 5, 202-205.

Carey, S., and Xu, F. (2001). Infants' knowledge of objects: beyond object files and object tracking. Cognition 80 , 179-213.

Cashon, C. H., and Cohen, L. B. (2000). Eight-month-old infants' perception of possible and impossible events. Infancy 1, 429-446.

Chawla, D., Rees, G., and Friston, K. J. (1999). The physiological basis of attentional modulation in extrastriate visual areas. Nat. Neurosci. 2, 671-676.

Cheries, E. W., Mitroff, S. R., Wynn, K., and Scholl, B. J. (in press). Do the same principles constrain persisting object representations in infant cognition and adult perception?: The cases of continuity and cohesion. In The Origins of Object Knowledge, B. Hood and L. Santos, eds (Oxford University Press).

Cheries, E. W., Wynn, K., and Scholl, B. J. (2006). Interrupting infants' persisting object representations: an objectbased limit? Dev. Sci. 9, F50-F58.

Chiang, W. C., and Wynn, K. (2000). Infants' tracking of objects and collections. Cognition 77, 169-195.

Cohen, L. B. (1976). Habituation of infant visual attention. In Habituation: Perspectives From Child Development, Animal Behavior, and Neurophysiology, T.J. Tighe and R. N. Leaton, eds (Hillsdale, NJ, Lawrence Erlbaum), pp. 207-238.

Cohen, L. B., DeLoache, J. S., and Pearl, R. A. (1977). An examination of interference effects in infants' memory for faces. Child Dev. 48, 88-96.

Cohen, L. B., and Gelber, E. R. (1975). Infant visual memory. In Infant Perception, L. Cohen and P. Salapatek, eds (New York, Academic Press), pp. 347-403.

Cohen, L. B., and Marks, K. S. (2002). How infants process addition and subtraction events. Dev. Sci. 5, 186-201.

Dehaene-Lambertz, G., Dehaene, S., Anton, J. L., Campagne, A., Ciuciu, P., Dehaene, G. P., Denghien, I., Jobert, A., LeBihan, D., and Sigman, M. (2006). Functional segregation of cortical language areas by sentence repetition. Hum. Brain Mapp. 27, 360-371.

Desimone, R. (1996). Neural mechanisms for visual memory and their role in attention. Proc. Natl. Acad. Sci. U.S.A. 93, 13494-13499.

Desimone, R., and Duncan, J. (1995). Neural mechanisms of selective visual attention. Annu. Rev. Neurosci. 18, 193-222.

Dobbins, I.G.,Schnyer,D.M.,Verfaellie, M., and Schacter, D. L. (2004). Cortical activity reductions during repetition priming can result from rapid response learning. Nature 428, 316-319.

Dolan, R.J., Fink, G. R., Rolls, E., Booth, M., Holmes, A., Frackowiak, R. S., and Friston, K. J. (1997). How the brain learns to see objects and faces in an impoverished context. Nature 389, 596-599.

Eger, E., Henson, R. N., Driver, J., and Dolan, R. J. (2004). BOLD repetition decreases in object-responsive ventral visual areas depend on spatial attention. J. Neurophysiol. 92, 1241-1247.
Epstein, R., and Kanwisher, N. (1998). A cortical representation of the local visual environment. Nature 392, 598-601.

Fagan, J. F. (1970). Memory in the infant. J. Exp. Child Psychol. 9, 217-226.

Fagan, J. F. (1972). Infants' recognition memory for faces. J. Exp. Child Psychol. $14,453-476$.

Fantz, R. L. (1964). Visual experience in infants: decreased attention to familiar patterns relative to novel ones. Science $146,668-670$.

Feigenson, L., Carey, S., and Spelke, E. (2002). Infants' discrimination of number vs. continuous extent. $\operatorname{Cog} n$. Psychol. 44, 33-66.

Fiser, J., and Aslin, R. N. (2002). Statistical learning of new visual feature combinations by infants. Proc. Natl. Acad. Sci. U.S.A. 99, 15822-15826.

Flombaum, J. I., Scholl, B. J., and Santos, L. R. (in press). Spatiotemporal priority as a fundamental principle of object persistence. In The Origins of Object Knowledge. B. Hood and L. Santos, eds (Oxford University Press).

Geva, R., Gardner, J. M., and Karmel, B.Z. (1999). Feeding-based arousal effects on visual recognition memory in early infancy. Dev. Psychol. 35, 640-650.

Gliga, T., and Dehaene-Lambertz, G. (2007). Development of a view-invariant representation of the human head. Cognition 102, 261-288.

Grill-Spector, K., Henson, R., and Martin, A. (2006). Repetition and the brain: neural models of stimulusspecific effects. Trends Cogn. Sci. 10, 14-23.

Grill-Spector, K., Kushnir, T., Edelman, S. Avidan, G., Itzchak, Y., and Malach, R. (1999). Differential processing of objects under various viewing conditions in the human lateral occipital complex. Neuron 24, 187-203.

Grill-Spector, K., Kushnir, T., Hendler, T. and Malach, R. (2000). The dynamics of object-selective activation correlate with recognition performance in humans. Nat. Neurosci. 3, 837-843.

Groves, P.M., and Thompson, R. F. (1970). Habituation: a dual-process theory. Psychol. Rev. 77, 419-450.

Haith, M. M. (1998). Who put the cog in infant cognition? Is rich interpretation too costly. Infant. Behav. Dev. 21, 167-179.

Henson, R., Shallice, T., and Dolan, R. (2000). Neuroimaging evidence for dissociable forms of repetition priming. Science 287, 1269-1272.

Henson, R. N. (2003). Neuroimaging studies of priming. Prog. Neurobiol. 70, 53-81.

Henson, R. N., Rylands, A., Ross, E., Vuilleumeir, P., and Rugg, M. D.
(2004). The effect of repetition lag on electrophysiological and haemodynamic correlates of visual object priming. Neuroimage 21, 1674-1689.

Hubel, D. H., and Wiesel, T. N. (1977). Ferrier lecture. Functional architecture of macaque monkey visual cortex. Proc. R. Soc. Lond., B, Biol. Sci. 198, $1-59$.

Hunt, J. (1970). Attentional preference and experience: I. Introduction. J. Genet. Psychol. 117, 99-107.

Hunter, M. A., and Ames, E. W. (1988). A multifactor model of infant preferences for novel and familiar stimuli. Adv. Infancy Res. 5, 69-95.

Hunter, M.A., Ross, H.S., and Ames, E. W. (1982). Preferences for familiar or novel toys: effects of familiarization time in 1-year-olds. Dev. Psychol. 18, 519-529.

James, T. W., and Gauthier, I. (2006). Repetition-induced changes in BOLD response reflect accumulation of neural activity. Hum. Brain Mapp. 27, 37-46.

Jenkins, A. C., Macrae, C. N., and Mitchell, J. P. (2008). Repetition suppression of ventromedial prefrontal activity during judgments of self and others. Proc. Natl. Acad. Sci. 105, 4507-4512.

Johnson, M. H. (2000). Functional brain development in infants: elements of an interactive specialization framework. Child Dev. 71, 75-81.

Kagan, J., and Lewis, M. (1965). Studies of attention in the human infant. Merrill Palmer Q. 11, 95-127.

Kanwisher, N., McDermott, J., and Chun, M. M. (1997). The fusiform face area: a module in human extrastriate cortex specialized for face perception. J. Neurosci. 17, 4302-4311.

Kourtzi, Z., Betts, L. R., Sarkheil, P., and Welchman, A. E. (2005). Distributed neural plasticity for shape learning in the human visual cortex. PLoS Biol. 3, e204.

Kourtzi, Z., and Huberle, E. (2005). Spatiotemporal characteristics of form analysis in the human visual cortex revealed by rapid event-related fMRI adaptation. Neuroimage 28, 440-452.

Kourtzi, Z., and Kanwisher, N. (2001). Representation of perceived object shape by the human lateral occipital complex. Science 293, 1506-1509.

Krekelberg, B., Boynton, G. M., and van Wezel, R. J. (2006). Adaptation: from single cells to BOLD signals. Trends Neurosci. 29, 250-256.

Leber, A. B., Turk-Browne, N. B., and Chun,M.M. (2008). Neural predictors of moment-to-moment fluctuations in cognitive flexibility. Proc. Natl. Acad. Sci. U.S.A. 105, 13592-13597. 
Li, L., Miller, E. K., and Desimone, R. (1993). The representation of stimulus familiarity in anterior inferior temporal cortex. J. Neurophysiol. 69, 1918-1929.

Maccotta, L., and Buckner, R. L. (2004). Evidence for neural effects of repetition that directly correlate with behavioral priming. J. Cogn. Neurosci. 16, 1625-1632.

Malach, R., Reppas, J. B., Benson, R. R., Kwong, K. K., Jiang, H., Kennedy, W. A., Ledden, P. J., Brady, T. J., Rosen, B. R., and Tootell, R. B. (1995). Objectrelated activity revealed by functional magnetic resonance imaging in human occipital cortex. Proc. Natl. Acad. Sci. U.S.A. 92, 8135-8139.

Manns, J. R., Stark, C. E., and Squire, L. R. (2000). The visual paired-comparison task as a measure of declarative memory. Proc. Natl. Acad. Sci. 97, 12375-12379.

McCarthy, G., Puce, A., Gore, J. C., and Allison, T. (1997). Face-specific processing in the human fusiform gyrus. J. Cogn. Neurosci. 9, 605-610.

McKee, R. D., and Squire, L. R. (1993). On the development of declarative memory. J. Exp. Psychol. Learn. Mem. Cogn. 19, 397-404.

Miller, E. K., and Desimone, R. (1994). Parallel neuronal mechanisms for short-term memory. Science 263, 520-522.

Murray, S.O., Olman, C.A., and Kersten, D. (2006). Spatially specific fMRI repetition effects in human visual cortex. J. Neurophysiol. 95, 2439-2445.

Murray, S. O., and Wojciulik, E. (2004). Attention increases neural selectivity in the human lateral occipital complex. Nat. Neurosci. 7, 70-74.

Nelson, C. A. (1995). The ontogeny of human memory: a cognitive neuroscience perspective. Dev. Psychol. 31, 723-738.

O'Craven, K. M., Downing, P. E., and Kanwisher, N. (1999). fMRI evidence for objects as the units of attentional selection. Nature 401, 584-587.

Olson, G. M. (1976). An informationprocessing analysis of visual memory and habituation in infants. In Habituation: Perspectives From Child Development, Animal Behavior, and Neurophysiology, T. J. Tighe and R. N. Leaton, eds (Hillsdale, NJ, Lawrence Erlbaum), pp. 239-278.

Park, S., Intraub, H., Yi, D. -J., Widders, D., and Chun, M. M. (2007). Beyond the edges of a view: boundary extension in human scene-selective visual cortex. Neuron 54, 335-342.

Pessoa, L., McKenna, M., Gutierrez, E., and Ungerleider, L. G. (2002). Neural processing of emotional faces requires attention. Proc. Natl. Acad. Sci. U.S.A. 99, 11458-11463.
Piaget, J. (1954). The Construction of Reality in the Child. New York, Basic Books.

Piazza, M., Izard, V., Pinel, P., Le Bihan, D., and Dehaene, S. (2004). Tuning curves for approximate numerosity in the human intraparietal sulcus. Neuron 44, 547-555.

Puce, A., Allison, T., and McCarthy, G. (1999). Electrophysiological studies of human face perception: III. Effects of top-down processing on face-specific potentials. Cereb. Cortex 9, 445-458.

Quinn, P. C. (1987). The categorical representation of visual pattern information by young infants. Cognition 27, 145-179.

Richards, J. E. (1997). Effects of attention on infants' preference for briefly exposed visual stimuli in the pairedcomparison recognition-memory paradigm. Dev. Psychol. 33, 22-31.

Roder, B. J., Bushnell, E. W., and Sasseville, A. M. (2000). Infants' preferences for familiarity and novelty during the course of visual processing. Infancy 1, 491-507.

Rose, S. A., Gottfried, A. W., MelloyCarminar, P., and Bridger, W. H. (1982). Familiarity and novelty preferences in infant recognition memory: implications for information processing. Dev. Psychol. 18, 704-713.

Rotshtein, P., Henson, R. N., Treves, A., Driver, J., and Dolan, R. J. (2005). Morphing Marilyn into Maggie dissociates physical and identity face representations in the brain. Nat. Neurosci. 8, 107-113.

Ryan, J.D., Leung, G., Turk-Browne, N. B., and Hasher, L. (2007). Assessment of age-related changes in inhibition and binding using eye movement monitoring. Psychol. Aging 22, 239-250.

Sawamura, H., Orban, G.A., and Vogels, R. (2006). Selectivity of neuronal adaptation does not match response selectivity: a single-cell study of the FMRI adaptation paradigm. Neuron 49, 307-318.

Schacter, D. L., and Buckner, R. L. (1998). Priming and the brain. Neuron 20 185-195.

Schacter, D. L., Wig, G. S., and Stevens, W. D. (2007). Reductions in cortical activity during priming. Curr. Opin. Neurobiol. 17, 171-176.

Scholl, B. J., and Leslie, A. M. (1999). Explaining the infant's object concept: beyond the perception/cognition dichotomy. In What is Cognitive Science?, E. Lepore and Z. Pylyshyn, eds (Oxford, Blackwell), pp. 26-73.

Schoner, G., and Thelen, E. (2006). Using dynamic field theory to rethink infant habituation. Psychol. Rev. 113, 273-299.

Schott, B. H., Henson, R. N., RichardsonKlavehn, A., Becker, C., Thoma, V.,
Heinze, H. J., and Duzel, E. (2005) Redefining implicit and explicit memory: the functional neuroanatomy of priming, remembering, and control of retrieval. Proc. Natl. Acad. Sci. 102, 1257-1262.

Sirois, S., and Mareschal, D. (2002) Models of habituation in infancy. Trends Cogn. Sci. 6, 293-298.

Snyder, K. A., Blank, M. P., and Marsolek, C. J. (2008). What form of memory underlies novelty preferences? Psychon. Bull. Rev. 15, 315-321.

Snyder, K. A., and Keil, A. (in press). Repetition suppression of induced gamma activity predicts enhanced orienting toward a novel stimulus in 6-month-old infants. J. Cogn. Neurosci.

Southgate, V., Csibra, G., Kaufman, J., and Johnson, M. H. (2008). Distinct processing of objects and faces in the infant brain. J. Cogn. Neurosci. 20 741-749.

Spelke, E. S. (1988). Where perceiving ends and thinking begins: the apprehension of objects in infancy. In Perceptual Development in Infancy, A. Yonas, ed. (Hillsdale, Erlbaum), pp. 197-234.

Spelke, E.S. (1998). Nativism, empiricism, and the origins of knowledge. Infant Behav. Dev. 21, 181-200.

Spelke, E. S., Breinlinger, K., Macomber, J., and Jacobson, K. (1992). Origins of knowledge. Psychol. Rev. 99, 605-632.

Tanaka, K. (1996). Inferotemporal cortex and object vision. Annu. Rev. Neurosci. 19, 109-139.

Thompson-Schill, S. L., D’Esposito, M. and Kan, I. P. (1999). Effects of repetition and competition on activity in left prefrontal cortex during word generation. Neuron 23, 513-522.

Turk-Browne, N. B., Yi, D. J., and Chun, M. M. (2006). Linking implicit and explicit memory: common encoding factors and shared representations. Neuron 49, 917-927.

Turk-Browne, N. B., Yi, D. J., Leber, A. B. and Chun, M. M. (2007). Visual quality determines the direction of neural repetition effects. Cereb. Cortex 17, 425-433.

van Turennout, M., Ellmore, T., and Martin,A. (2000).Long-lasting cortical plasticity in the object naming system. Nat. Neurosci. 3, 1329-1334.

Vuilleumier, P., Schwartz, S., Duhoux, S., Dolan, R. J., and Driver, J. (2005). Selective attention modulates neural substrates of repetition priming and "implicit" visual memory: suppressions and enhancements revealed by fMRI. J. Cogn. Neurosci. 17 1245-1260.

Wang, S., and Baillargeon, R. (2006). Infants' physical knowledge affects their change detection. Dev. Sci. 9 173-181.

Wang, S., Baillargeon, R., and Brueckner, L. (2004). Young infants' reasoning about hidden objects: evidence from violation-of-expectation tasks with test trials only. Cognition 93, 167-198.

Wang, S., Baillargeon, R., and Paterson, S. (2005). Detecting continuity violations in infancy: a new account and new evidence from covering and tube events. Cognition 95, 129-173.

Westermann, G., Mareschal, D., Johnson, M. H., Sirois, S., Spratling, M. W., and Thomas, M. S. (2007). Neuroconstructivism. Dev. Sci. 10, 75-83.

Wetherford, M. J., and Cohen, L. B. (1973). Developmental changes in infant visual preferences for novelty and familiarity. Child Dev. 44, 416-424.

Wiebe, S. A., Cheatham, C. L., Lukowski, A. F., Haight, J. C., Muehleck, A. J., and Bauer, P. J. (2006). Infants' ERP responses to novel and familiar stimuli change over time: implications for novelty detection and memory. Infancy 9, 21-44.

Wig, G. S., Grafton, S. T., Demos, K. E., and Kelley, W. M. (2005). Reductions in neural activity underlie behavioral components of repetition priming. Nat. Neurosci. 8, 1228-1233.

Wiggs, C. L., and Martin, A. (1998). Properties and mechanisms of perceptual priming. Curr. Opin. Neurobiol. 8, 227-223.

Winston, J. S., Henson, R. N., FineGoulden, M. R., and Dolan, R. J. (2004). fMRI-adaptation reveals dissociable neural representations of identity and expression in face perception. J. Neurophysiol. 92, 1830-1839.

Wynn, K. (1992). Addition and subtraction by human infants. Nature 358 , 749-750.

Wynn, K. (2002). Do infants have numerical expectations or just perceptual preferences? Dev. Sci. 5, 207-209.

Xu, Y., Turk-Browne, N. B., Chun, M. M. (2007). Dissociating task performance from $\mathrm{AMRI}$ repetition attenuation in ventral visual cortex. J. Neurosci. 27, 5981-5985.

Yi, D. J., and Chun, M. M. (2005). Attentional modulation of learningrelated repetition attenuation effects in human parahippocampal cortex. J. Neurosci. 25, 3593-3600.

Yi, D. J., Turk-Browne, N. B., Flombaum, J. I., Kim, M. S., Scholl, B. J., and Chun, M. M. (2008). Spatiotemporal object continuity in human ventral visual cortex. Proc. Natl. Acad. Sci. U.S.A. 105, 8840-8845.

Yi, D. J., Woodman, G. F., Widders, D., Marois, R., and Chun, M. M. (2004). Neural fate of ignored stimuli: dissociable effects of perceptual and 
working memory load. Nat. Neurosci. 7, 992-996.

Young-Browne, G., Rosenfeld, H. M., and Horowitz, F. D. (1977). Infant discrimination of facial expressions. Child Dev. 48, 555-562.

Zago, L., Fenske, M. J., Aminoff, E., and Bar, M. (2005). The rise and fall of priming: how visual exposure shapes cortical representations of objects. Cereb. Cortex 15, 1655-1665.

Conflict of Interest Statement: The authors declare that the research was conducted in the absence of any commercial or financial relationships that could be construed as a potential conflict of interest.
Received: 27 August 2008; paper pending published: 14 October 2008; accepted: 15 October 2008; published online: 02 December 2008

Citation: Turk-Browne NB, Scholl BJ and Chun MM (2008) Babies and brains: habituation in infant cognition and functional neuroimaging.Front.Hum. Neurosci.(2008) 2:16. doi: 10.3389/neuro.09.016.2008
Copyright () 2008 Turk-Browne, Scholl and Chun. This is an open-access article subject to an exclusive license agreement between the authors and the Frontiers Research Foundation, which permits unrestricted use, distribution, and reproduction in any medium, provided the original authors and source are credited. 